\title{
Influence of protein concentration on the stability of oil-in-water emulsions formed with aggregated milk proteins during spray drying
}

\author{
A. Taneja $\cdot$ A. Ye $\cdot$ H. Singh
}

Received: 4 August 2014 / Revised: 28 October 2014 / Accepted: 25 November 2014 / Published online: 18 December 2014

C INRA and Springer-Verlag France 2014

\begin{abstract}
The ability of aggregated milk proteins to stabilize oil-in-water emulsions during the spray drying process is not well understood and has been highlighted in this study. We investigated the behavior of oil droplets in emulsions $(12.0 \%, w / w$, maltodextrin; $20.0 \%, w / w$, soya oil) stabilized with either milk protein concentrate (MPC) or calcium caseinate (CaCas) $(0.5-5.0 \%, w / w)$ before and after spray drying. In comparison with the respective parent emulsions, spray drying and redispersion caused a shift in the droplet size distribution toward larger values for emulsions made using low concentrations (0.5-2.0\%) of MPC or CaCas. However, the droplet size distribution was affected very slightly by spray drying when the protein concentration was between 3.0 and $5.0 \%$. The average droplet diameters of redispersed $\mathrm{CaCas}$ emulsions were noticeably larger than those of redispersed MPC emulsions. The unadsorbed protein concentrations in the parent emulsions were comparable for emulsions containing CaCas and MPC, except at $5.0 \%(w / w)$ when emulsions containing MPC had a higher concentration of unadsorbed protein in the bulk phase. The amount of extractable oil in MPC-containing powders decreased with an increase in the protein concentration in the emulsions prior to spray drying. However, the amount of extractable oil in CaCas emulsion powders was nearly twice that in MPC emulsion powders even at 5.0\% protein in the emulsion. The results obtained from this study clearly show that aggregated milk proteins are able to provide stability to oil droplets during the spray drying process, albeit a higher concentration of protein is required as compared to non-aggregated protein products.
\end{abstract}

Keywords Re-coalescence $\cdot$ Redispersion $\cdot$ Extractable oil $\cdot$ Droplet diameter

\section{Introduction}

Milk proteins are important components in a number of categories of processed food because of their excellent nutritional and functional properties. Many different

A. Taneja $\cdot$ A. Ye $\cdot$ H. Singh $(\bowtie)$

Riddet Institute, Massey University, Private Bag 11 222, Palmerston North 4442, New Zealand

e-mail: H.Singh@massey.ac.nz 
variations of milk protein products are now commercially available for use as food ingredients, e.g., caseins and caseinates, whey protein concentrates (WPCs) and whey protein isolates (WPIs), milk protein concentrates (MPCs), and other specifically designed products for particular applications (Ye 2011). The structure, conformation, and aggregation state of the protein molecules in milk protein products have a considerable impact on their ability to stabilize the oil droplets in oil-in-water emulsions (Euston and Hirst 1999; Faldt and Bergenstahl 1996; Singh and Ye 2009; Vega and Roos 2006). MPC products containing 56-82\% (w/w) protein are processed directly from skim milk by a combination of ultrafiltration and diafiltration. In these products, the whey proteins remain in their native form and the caseins are retained in micellar form, similar to their structures in milk (Dybowska 2008; Havea 2006; Singh and Ye 2009). In contrast, caseinates are manufactured by the acidification of milk to $\mathrm{pH} 4.6$ and separation of the casein curd. Following washing of the curd, the $\mathrm{pH}$ is adjusted to 7.0 with either sodium hydroxide or calcium hydroxide, resulting in sodium caseinate (NaCas) or calcium caseinate (CaCas), respectively (Srinivasan et al. 2003).

The exact state of the casein molecules in NaCas solutions is unknown; NaCas solution has been reported to contain a mixture of individual casein molecules and small aggregates with varying casein composition and with sizes around 10-30 nm (Farrer and Lips 1999; Lucey et al. 2000). CaCas consists of mixtures of casein aggregates of different sizes, many of which are much larger than those found in NaCas solutions (Srinivasan et al. 2003).

$\mathrm{NaCas}$ shows excellent emulsifying ability, and very stable emulsions can be made at a relatively low protein-to-oil ratio (about 1:50). A stable emulsion can be defined as one which is able to resist any change in its properties such as droplet size distribution and state of aggregation over the time scale of observation (Singh and Ye 2009; Vega and Roos 2006). In these emulsions, the saturation surface protein coverage value is about 2.0-3.0 mg.m $\mathrm{m}^{-2}$ (Dickinson 1999; Singh 2005; Singh and Ye 2009). In contrast, the emulsifying ability of "aggregated" milk protein products, such as MPC and CaCas, is much lower than that of whey protein or NaCas, under similar homogenization conditions. The surface protein coverage of emulsions formed with these products is much higher, generally in the range 5-20 mg. $\mathrm{m}^{-2}$, depending on the protein concentration (Euston and Hirst 1999; Srinivasan et al. 1999). Under similar homogenization conditions, MPC and CaCas emulsions exhibit larger average droplet diameters than NaCas emulsions (Euston and Hirst 1999). In MPC and CaCas emulsions, bridging flocculation occurs at low protein concentrations because of the sharing of protein aggregates by adjacent droplets. In addition, the spreading of protein at the droplet surface is limited because the aggregates are held together by calcium bonds and/or colloidal calcium phosphate (Euston and Hirst 1999; Ye 2011).

Taneja et al. (2013) reported that milk protein-based emulsions formed with $>2.0 \%$ protein (NaCas or whey proteins) remained stable during spray drying, as indicated by the change in the average droplet diameter after spray drying. This corresponded to a critical concentration of $\sim>1.0 \%$ unadsorbed protein in the bulk phase of the emulsions. However, below this critical unadsorbed protein concentration in the bulk phase of the emulsions, the average droplet diameter shifted toward a larger size range on spray drying (Taneja et al. 2013). Although the emulsifying and adsorption properties of MPC and CaCas in oil-in-water emulsions have been well documented (Euston and 
Hirst 1999; Ye 2011; Ye et al. 2000), their ability to stabilize oil droplets during spray drying has not been reported.

A key parameter in spray-dried powdered emulsions is the amount of free oil on the surface of the powder particles. This free oil on the surface determines the flowability and the wettability of the powder. As rapid and complete reconstitution is a key quality indication for consumers, an understanding of the mechanism of the migration of oil to the surface of the powder particle during drying will be very useful (Kim et al. 2009; Murrieta-Pazos et al. 2012). The objective of this work was to evaluate the stabilizing ability of aggregated milk protein products, i.e., MPC and CaCas, during the spray drying of oil-inwater emulsions and to investigate its impact on the surface composition of the final powder.

\section{Materials and methods}

\subsection{Materials}

Soya oil was obtained from Davis Trading Co. (Palmerston North, New Zealand) and maltodextrin (Fieldose $17 \mathrm{GV}$, DE 17, maize starch) was supplied by Penford Australia Ltd. (Lane Cove, NSW, Australia). CaCas (Alanate 380; $\approx 94 \%$ protein) and MPC (Alapro $4850 ; \approx 83 \%$ protein) were supplied by Fonterra Co-operative Group Ltd. (New Zealand). Sodium dodecyl sulphate (SDS) was obtained from Sigma-Aldrich NZ (Auckland, New Zealand). All other chemicals were of reagent grade and were purchased from Sigma-Aldrich NZ (Auckland, New Zealand).

\subsection{Emulsion preparation}

Emulsions were prepared according to the method described by Taneja et al. (2013). $\mathrm{CaCas}$ and MPC $(12 \%, w / w)$ were dissolved in water purified by reverse osmosis (RO) using an IKAMAG RET stirrer (Basic C, IKA-Werke, Staufen, Germany), and the solution was stirred for at least $6 \mathrm{~h}$ at room temperature to allow complete hydration. The $\mathrm{pH}$ of the solution was then adjusted to 7.0 using $1 \mathrm{M} \mathrm{HCl}$ if required. Similarly, maltodextrin solution $(40 \%, w / w)$ was prepared using the same stirring equipment, with stirring for 60 min until the powder had dissolved completely. Soya oil at $20 \%(w / w)$ was blended with the aqueous solutions of protein and maltodextrin using an UltraTurrax T-25 hand-held homogenizer (Janke \& Kunkel, Staufen, Germany) operated at 13,500 rev. $\mathrm{min}^{-1}$ for $2 \mathrm{~min}$. The resulting primary emulsion was further homogenized at $60 \mathrm{MPa}$ for one cycle using a Microfluidizer (M-110P, Y-type interaction chamber, Microfluidics, Newton, MA, USA). About $500 \mathrm{~mL}$ of emulsion was prepared for each variation for spray drying purposes. Emulsions were prepared in duplicate.

\subsection{Spray drying}

All emulsions were spray dried using a Buchi bench-top spray drier (B-290, Buchi Labortechnik AG, Flawil, Switzerland) and standard glassware in co-current configuration and fitted with a two-fluid nozzle (nozzle cap $1.4 \mathrm{~mm}$ ). The feed rate of the emulsion varied from 300 to $400 \mathrm{~mL} \cdot \mathrm{h}^{-1}$. The inlet temperature varied between 140 and $165{ }^{\circ} \mathrm{C}$, and the outlet temperature was $65^{\circ} \mathrm{C}$. The resulting powders were 
collected in air-tight containers, allowed to cool to ambient temperature, and then stored at $4{ }^{\circ} \mathrm{C}$ for up to 6 months.

\subsection{Determination of average oil droplet size}

The average diameters of the oil droplets for both the parent emulsions and the redispersed emulsions were determined by the laser light scattering method as described by Jafari et al. (2007) and Ye (2011) using a Mastersizer E (Malvern Instruments, Malvern, Worcestershire, UK). Redispersed emulsions were prepared by reconstituting the dried powder in $\mathrm{RO}$ water to obtain the same total solids content as the parent emulsion. Powder was slowly added to RO water and the dispersions were stirred for at least $30 \mathrm{~min}$ before particle size measurements. In some cases, the emulsions were dispersed in $2.0 \%(w / w)$ SDS before the measurements. The comparison of the size distributions in RO water alone and with added SDS indicated flocculation/aggregation during emulsification/redispersion (Sanchez and Patino 2005). The droplet diameters were calculated as the averages of duplicate measurements.

\subsection{Total unadsorbed protein concentration}

The total unadsorbed protein concentration was determined using the method of Ye (2008) with some modifications. Emulsions (30 g) were centrifuged at 45,000 $\times \mathrm{g}$ for $45 \mathrm{~min}$ at $20^{\circ} \mathrm{C}$ in a temperature-controlled ultracentrifuge (Sorvall RC5C, DuPont Co., Wilmington, DE, USA), and the subnatants were carefully removed using a syringe. The cream layer was dispersed in RO water and re-centrifuged at $45,000 \times \mathrm{g}$ for $45 \mathrm{~min}$. Again, the subnatant and the cream layer were collected carefully. Each subnatant was filtered sequentially through 0.45 and $0.22 \mu \mathrm{m}$ filters (Millipore Corporation, Billerica, MA, USA). The filtrates were analyzed separately for total protein using the Kjeldahl method (1026 Distilling Unit and 1007 Digestor Block, Foss Tecator AB, Höganäs, Sweden). A factor of 6.38 was used to convert nitrogen to protein content. The total unadsorbed protein concentration (\%) was calculated by adding the percentage protein concentrations in each of the subnatants collected after centrifugation.

\subsection{Extractable oil}

The extractable oil content of the powdered emulsions was determined using solvent (petroleum ether, boiling point $40-60^{\circ} \mathrm{C}$ ) extraction, as described by Kim et al. (2005). One gram of powder was carefully weighed on to a filter paper (No. 4 Whatman, Maidstone, Kent, UK) and was washed four times with $5 \mathrm{~mL}$ of petroleum ether. The mixture of solvent and oil was collected in a conical flask, evaporated over a steam bath, and left overnight in the fume hood to allow further evaporation of the remaining ether. The flask was then placed in a $70^{\circ} \mathrm{C}$ oven for $1 \mathrm{~h}$ and then cooled in a desiccator. The amount of extracted oil was recorded as milligrams of free oil per gram of powder.

\subsection{Confocal scanning laser microscopy}

Confocal scanning laser microscopy (Leica DM6000B, Heidelberg, Germany) of emulsions and powders was carried out as described by Taneja et al. (2013). A 63 X 
oil immersion objective with an excitation wavelength of $488 \mathrm{~nm}$ from an argon laser was used for microscopy of emulsions. About $500 \mu \mathrm{L}$ of emulsion (both parent and redispersed) was taken in an Eppendorf tube, $10 \mu \mathrm{L}$ of Nile Blue (fluorescent dye, $0.1 \%, w / v$, in RO water) was added, and the sample was gently shaken. A volume of $60 \mu \mathrm{L}$ from this sample was placed on a concave microscope slide. A cover slip was carefully placed, and the sample was observed under the microscope. For powder samples, the fluorescent dyes were dissolved in the oil phase (Nile Blue, $0.02 \%, w / w$ ) and the protein solution (Fast green, $0.01 \%, w / w$ ), followed by emulsion preparation as described in Section 2.2. The resulting powder was carefully mounted on to a concave microscope slide using DPX (non-aqueous mounting medium). A cover slip was placed carefully on top, avoiding air bubbles. A $100 \mathrm{X}$ oil immersion objective with sequential excitation wavelength of $488 \mathrm{~nm}$ from an argon laser and of $633 \mathrm{~nm}$ from a helium-neon laser was used. All images were acquired at $20^{\circ} \mathrm{C}\left( \pm 2{ }^{\circ} \mathrm{C}\right)$.

\subsection{Scanning electron microscopy}

To investigate the morphological and structural properties of the powdered emulsions, an FEI Quanta 200 scanning electron microscope (FEI Electron Optics, Eindhoven, Netherlands) was used. The samples were placed on the scanning electron microscopy stubs using two-sided adhesive tape (Scotch, 3 M NZ, Auckland, New Zealand). The specimens were subsequently coated with gold using a sputter coater (Balzers SCD 050 Sputter Coater, Balzers, Liechtenstein). The coated samples were then analyzed using the microscope operating at an accelerating voltage of $20 \mathrm{kV}$. Micrographs, representing the microstructure of the powdered emulsions, were obtained using the FEI Quanta software installed on the PC connected to the system.

\section{Results}

\subsection{Droplet size distributions}

The droplet size distributions of emulsions $(12.0 \%, w / w$, maltodextrin; $20.0 \%, w / w$, soya oil) containing MPC or CaCas at concentrations from 0.5 to $5.0 \%(w / w)$ before and after spray drying are shown in Figs. 1 and 2, respectively. The size distributions were monomodal for all parent emulsions (before drying) containing MPC and shifted toward a smaller size range as the concentration of MPC in the bulk phase was increased from 0.5 to $2.0 \%(w / w)$. There was no noticeable change in the distribution when the concentration of MPC was increased further from 2.0 to $5.0 \%$ ( $w / w$, Fig. 1).

When the MPC emulsions were spray dried and then redispersed in RO water, for emulsions containing $\leq 2.0 \%(w / w)$ MPC, the size distributions shifted toward a larger size range compared with the parent emulsion at the corresponding protein concentration (Fig. 1). However, emulsions containing $\geq 3.0 \%(w / w)$ MPC showed good stability during spray drying because their size distributions were very similar before and after spray drying (Fig. 1).

The parent emulsions prepared using CaCas had bimodal size distributions that were noticeably broader than those of the parent MPC emulsions. As the CaCas concentration in the emulsion was increased from 0.5 to $5.0 \%(w / w)$, the size distribution shifted 

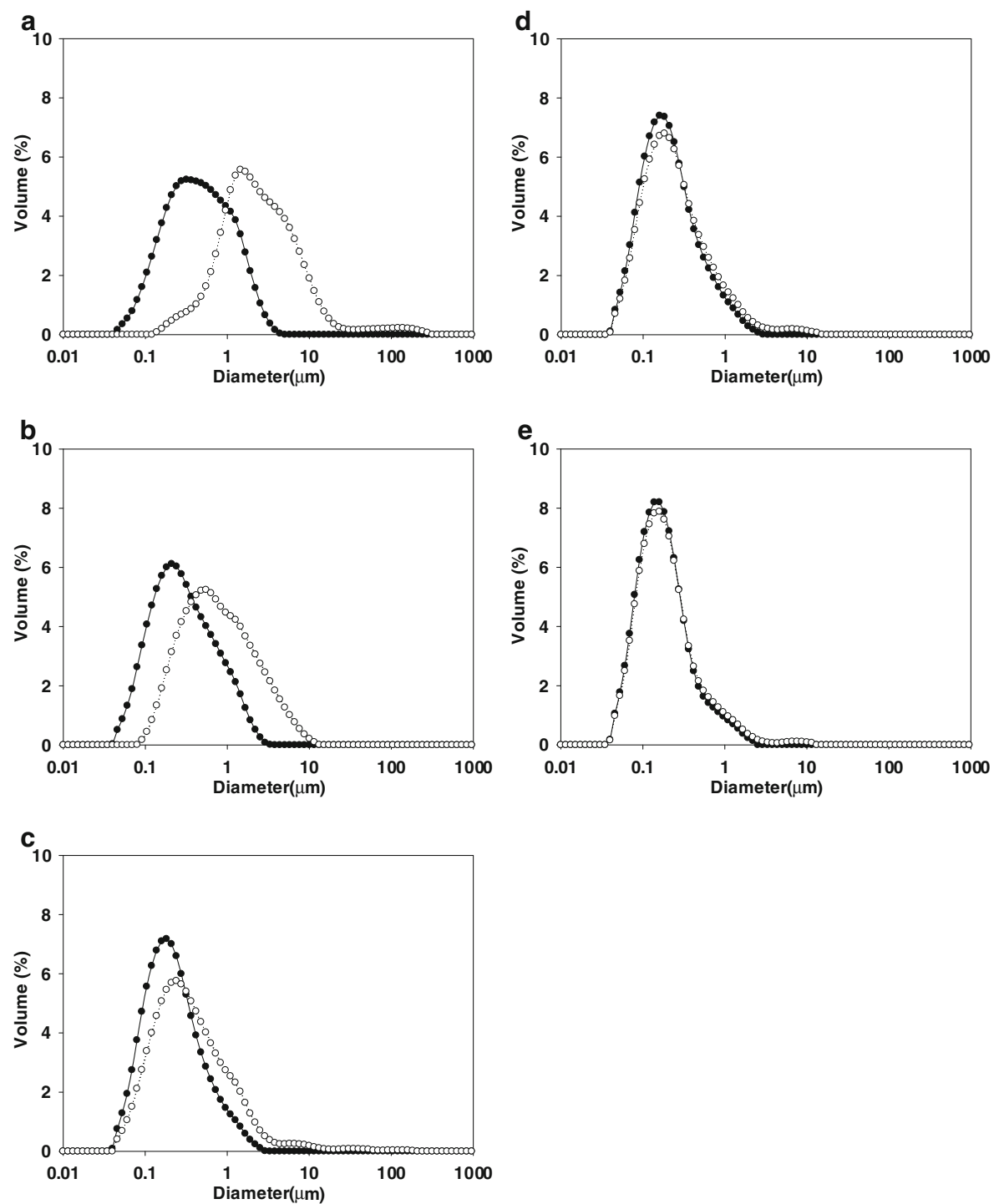

Fig. 1 Droplet size distributions of emulsions $(0.5-5.0 \%, w / w$, milk protein concentrate, $20.0 \%, w / w$, soya oil, $12.0 \%, w / w$, maltodextrin) before spray drying (black circle) and after redispersion (white circle): a $0.5 \%$, b $1.0 \%$, c $2.0 \%$, d $3.0 \%$, e $5.0 \%$ protein

toward a smaller size range (Fig. 2). After spray drying, all the redispersed emulsions made with CaCas had multimodal distributions that were shifted noticeably toward a larger size range because of the spray drying (Fig. 2). This shift toward a larger size range was greatest at $\leq 1.0 \%(w / w)$ CaCas in the emulsion. From these size distribution profiles, only the emulsion prepared with $5.0 \%(w / w)$ CaCas showed good redispersion, i.e., no significant change in size distribution after spray drying.

The changes in average droplet size $\left(d_{43}\right)$ of the MPC and CaCas emulsions upon spray drying are shown in Fig. 3 . The $d_{43}$ values of the redispersed emulsions made with $\leq 1.0 \%(w / w)$ MPC or CaCas were considerably higher than those of the parent 

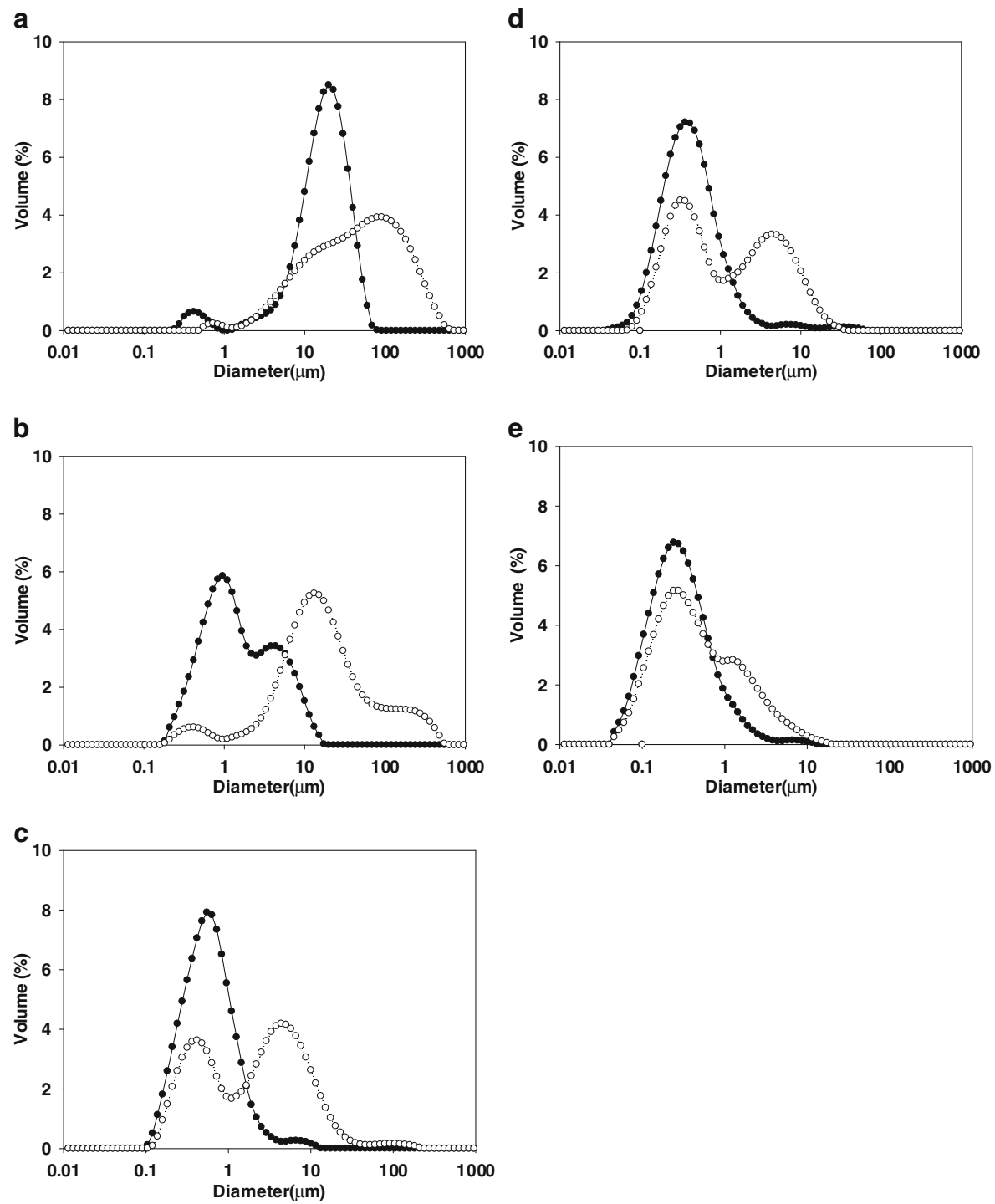

Fig. 2 Droplet size distributions of emulsions $(0.5-5.0 \%, w / w$, calcium caseinate, $20.0 \%$, w/w, soya oil, $12.0 \%, w / w$, maltodextrin) before spray drying (black circle) and after redispersion (white circle): a $0.5 \%$, b $1.0 \%$, c $2.0 \%$, d $3.0 \%$, e $5.0 \%$ protein

emulsions. When these emulsions were diluted (1:1) with 2.0\% (w/w) SDS solution (which breaks hydrophobic interactions and displaces protein molecules from the oilwater interface), there was a significant reduction in $d_{43}$, which indicated that the spray drying had caused flocculation and coalescence of the emulsion droplets. This was also visually evident in the confocal micrographs of the emulsion containing $0.5 \%(w / w)$ $\mathrm{CaCas}$, as shown in Fig. 4. According to the micrographs, droplet coalescence seems to be the dominating phenomenon. There was no change in the $d_{43}$ values when the redispersed emulsions made with $\geq 3.0 \%(w / w)$ MPC or CaCas were diluted with $2.0 \%$ $(w / w)$ SDS solution (Fig. 3). 

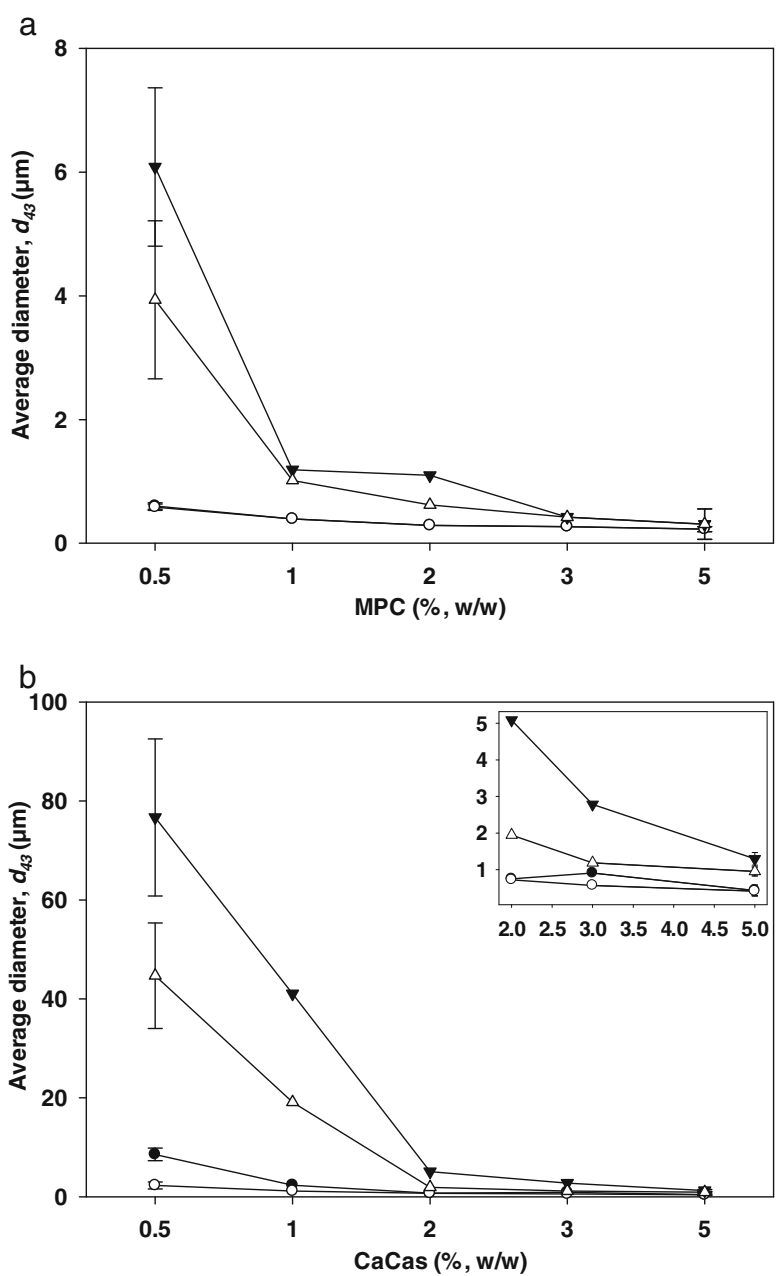

Fig. 3 Average droplet diameter $\left(d_{43}\right)$ values of parent (black circle) and redispersed (black pointing-down triangle) emulsions containing $20.0 \%(w / w)$ soya oil, $12.0 \%(w / w)$ maltodextrin, and $0.5-5.0 \%(w / w)$ milk protein concentrate (MPC) (a) or calcium caseinate (CaCas) (b). The parent (white circle) and redispersed (white triangle) emulsions were also diluted $(1: 1)$ with $2.0 \%(w / w)$ sodium dodecyl sulphate solution. Each data point is the average of two determinations on separate emulsions

It should be noted that the $d_{43}$ values of the redispersed emulsions containing CaCas were at least three times larger than those of the redispersed emulsions containing MPC at any given protein concentration. For example, at $1.0 \%(w / w)$ protein in the emulsions, the $d_{43}$ values of the redispersed emulsions containing MPC and CaCas were approximately $1 \mu \mathrm{m}$ and approximately $20 \mu \mathrm{m}$, respectively.

\subsection{Total unadsorbed protein}

In our previous study, we suggested that the total unadsorbed protein in the bulk phase plays a crucial role in the stability of the oil droplets during spray drying (Taneja et al. 2013). Therefore, we measured the total concentration of unadsorbed protein in the 

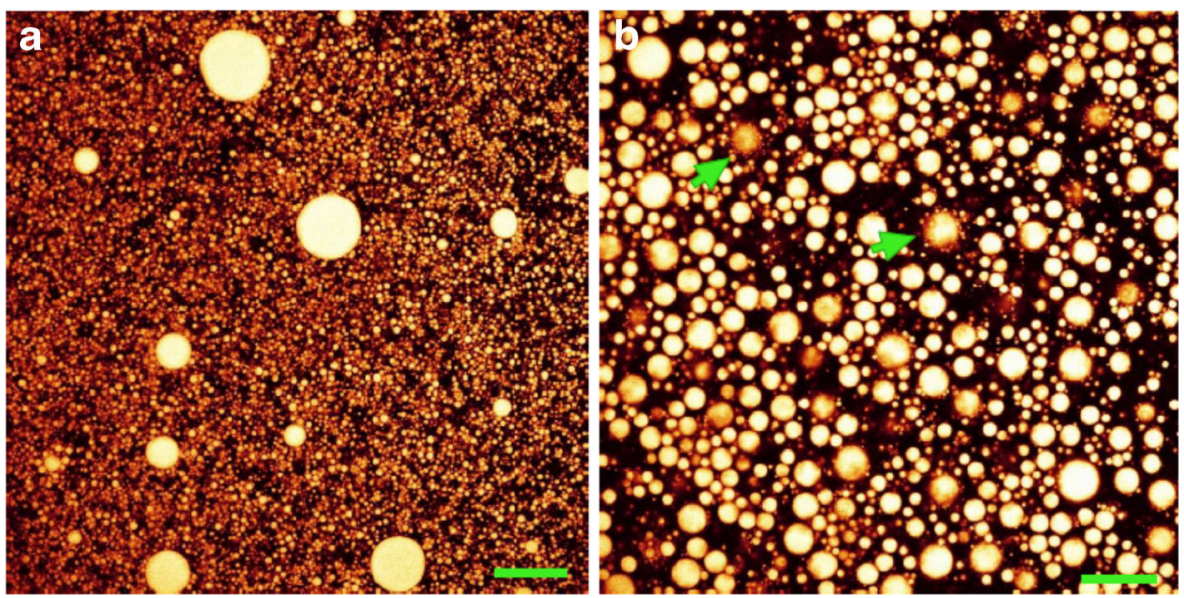

Fig. 4 Confocal micrographs of emulsions $(0.5 \%, w / w$, calcium caseinate, $20.0 \%, w / w$, soya oil, $12.0 \%, w / w$, maltodextrin) stained with Nile blue: a parent emulsion; b redispersed emulsion after spray drying. The scale on the micrographs represents $50 \mu \mathrm{m}$. Arrows illustrate bridging flocculation (b)

bulk phase of the parent emulsions stabilized by both MPC and CaCas. The amounts of unadsorbed protein in the bulk phase of the parent emulsions were comparable at all protein concentrations except at 5.0\% (w/w; Fig. 5). At 5.0\% protein, the amounts of protein that were free in the bulk phase were about 3.0\% for the MPC-stabilized emulsion and about $2.0 \%$ for the CaCas-stabilized emulsion.

\subsection{Extractable oil}

The extractable oil contents (mg. $\mathrm{g}^{-1}$ ) of the spray-dried emulsions stabilized with MPC and $\mathrm{CaCas}$ are shown in Fig. 6 as a function of protein concentration $(0.5-5.0 \%, w / w)$. For the emulsions stabilized with MPC, the amount of extractable oil decreased as the MPC concentration in the powder was increased up 3.0\% $(w / w)$, with no further change between 3.0 and 5.0\% (w/w) MPC. There was a similar trend for the emulsions stabilized with CaCas; however, the amounts of extractable oil were much higher in the CaCas-containing powders than in the MPC-containing powders. For both emulsions, the greatest amount of extractable oil was observed at the lowest protein concentration, i.e., $0.5 \%(w / w)$.

Fig. 5 Unadsorbed protein $(\%, w / w)$ in the bulk phase of the parent emulsions containing either milk protein concentrate (black circle) or calcium caseinate (white circle) as a function of the total protein concentration. Each data point is the average of two determinations on separate emulsions

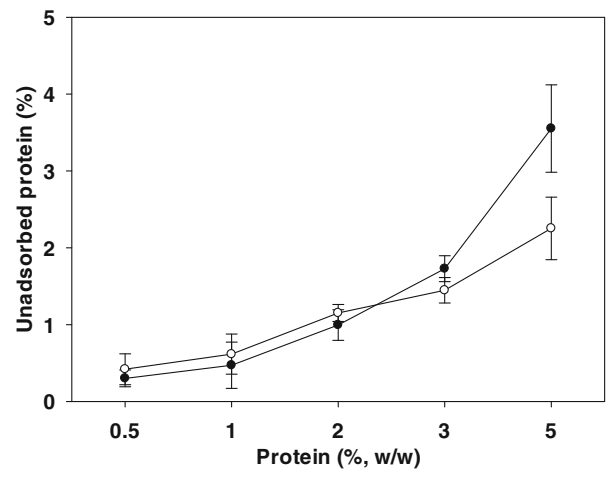

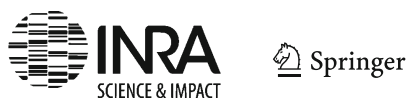


Fig. 6 Extractable oil (mg.g ${ }^{-1}$ )

for powders containing either milk protein concentrate (white circle) or calcium caseinate (black circle) as a function of protein concentration in the emulsions used to make the powders. Each data point is the average of two determinations on separate powders

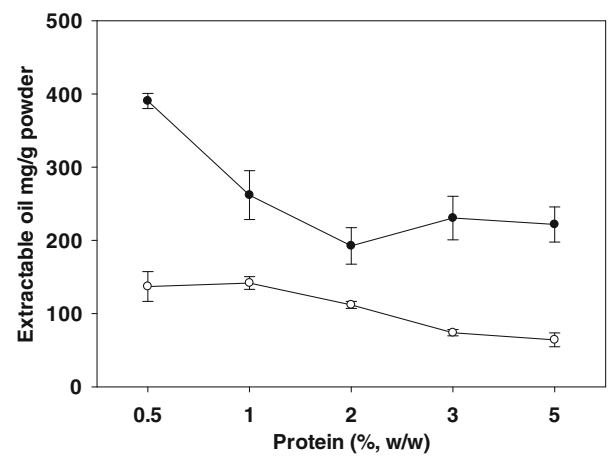

\subsection{Microstructure of spray-dried powder particles}

The electron micrographs of dried emulsions containing high and low concentrations of MPC and CaCas showed considerable differences in powder particle structure. At low MPC and CaCas concentrations, the powder particles appeared to be excessively agglomerated, almost giving a "molten" appearance, which possibly indicated high amounts of free oil on the surfaces of the powder particles (Fig. 7a, b). At high MPC and CaCas concentrations, the powder particles were observed to be mostly well separated and had considerable indentations. This appearance of dents or a shriveled texture was indicative of the formation of vapor bubbles inside the particles because of differences in the local vapor pressure. It has been suggested that, when powder particles reach parts of the drier in which the temperatures are relatively cooler, e.g., cyclones, the particles tend to deflate, resulting in a shriveled appearance (Nijdam and Langrish 2006).

The distribution of oil droplets within the powder particles containing MPC was studied using confocal scanning laser microscopy. The micrographs showed the presence of coalesced oil droplets (in red) in powdered emulsions containing $0.5 \%(w / w)$ MPC (Fig. 8a). In contrast, there were evenly distributed oil droplets inside the matrix of powder particles in the powdered emulsions containing 5.0\% (w/w) MPC (Fig. 8b). A large proportion of the confocal images (not shown) indicated an over representation of protein (green color) on the surface of the powder particles at all MPC concentrations $(0.5-5.0 \%, w / w)$.

\section{Discussion}

At a given protein concentration, the emulsifying ability of a protein is determined by the average droplet diameter of the droplets generated under defined homogenization conditions. Smaller droplet sizes are indicative of superior emulsifying ability (Euston and Hirst 1999). Milk proteins, either as individual molecules or as aggregated species, rapidly adsorb on to the surface of the oil droplets during emulsification, providing stability to the droplet. A number of factors affect the adsorption of protein on to the oil-water interface during homogenization, including protein concentration, oil volume, and the state of protein aggregation (Singh and Ye 2009). 

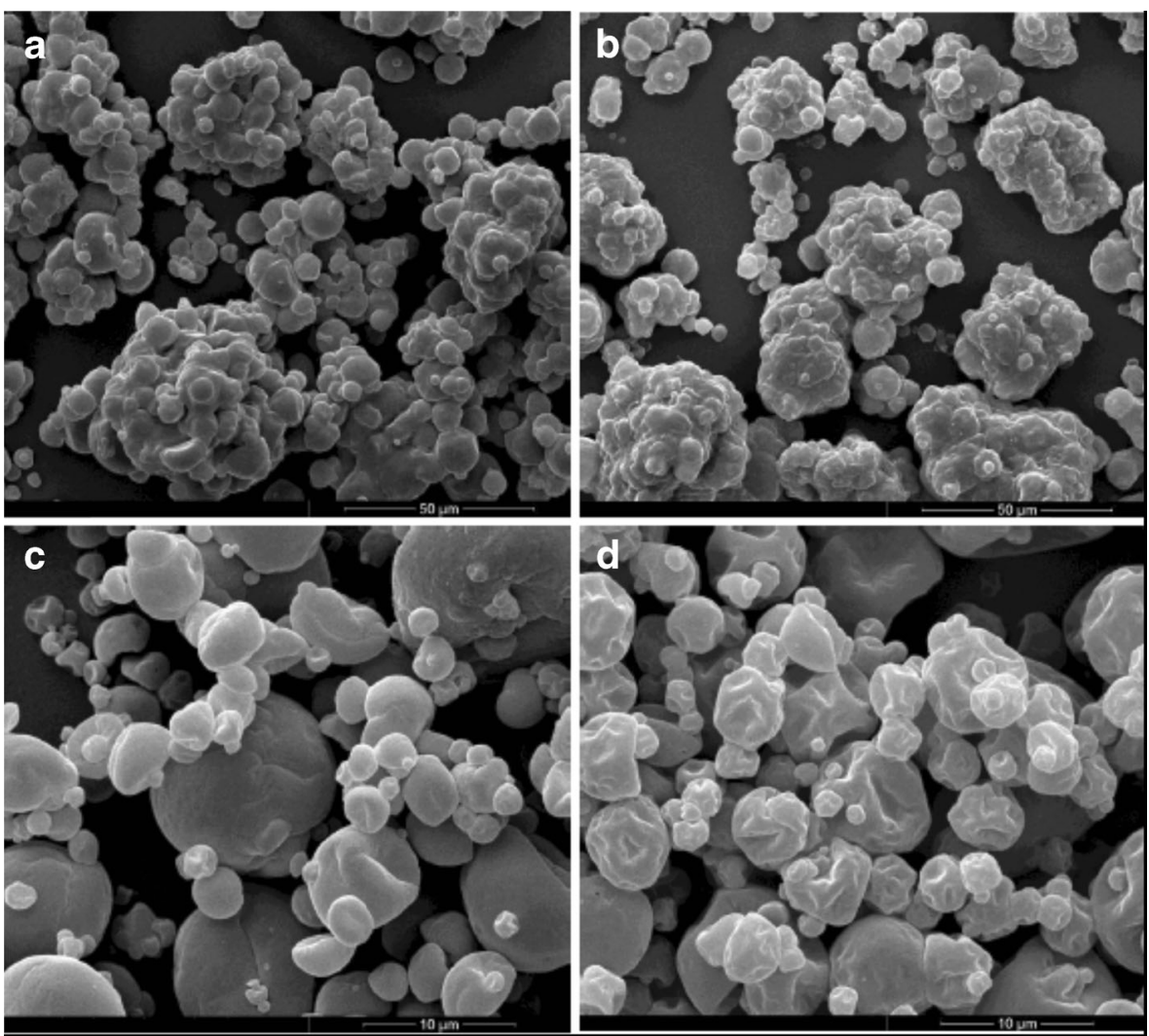

Fig. 7 Scanning electron micrographs of emulsion powder particles $(20.0 \%, w / w$, soya oil, $12.0 \%, w / w$, maltodextrin): a $0.5 \%(w / w)$ milk protein concentrate, b $0.5 \%(w / w)$ calcium caseinate, c $5.0 \%(w / w)$ milk protein concentrate, $\mathbf{d} 5.0 \%(w / w)$ calcium caseinate. Scale as shown on the micrographs

There are some important differences between MPC and CaCas in terms of the aggregation state and the composition of the proteins that are likely to influence their ability to form and stabilize oil-in-water emulsions. MPC contains both whey proteins and caseins, which are largely in the form of casein micelles (Euston and Hirst 1999; Kelly 2011). In addition, a small proportion of the protein material remains insoluble in water and consists mainly of large protein aggregates that are held together by noncovalent linkages. The majority of these insoluble aggregates have been reported to be composed of caseins and minor whey proteins (Havea 2006). In contrast, CaCas consists of various casein aggregates ranging in size from 20 to $1000 \mathrm{~nm}$ (Srinivasan et al. 2001). About 25\% of the casein in a CaCas solution appears to be highly aggregated, and these large aggregates are generated mainly via interactions of calcium with $\alpha_{\mathrm{s} 1}$ - and $\alpha_{\mathrm{s} 2}$-caseins (Euston and Hirst 1999; Srinivasan et al. 1999).

Because of the presence of whey proteins in addition to casein micelles, the emulsions formed with MPC had narrower size distributions at all protein concentrations than the emulsions formed with $\mathrm{CaCas}$. The casein aggregates in $\mathrm{CaCas}$ also have the ability to adsorb at the droplet surface, but not as efficiently as the monomeric caseins or the whey proteins. This would have a significant effect, especially at low protein concentrations, promoting coalescence and bridging flocculation, as the 

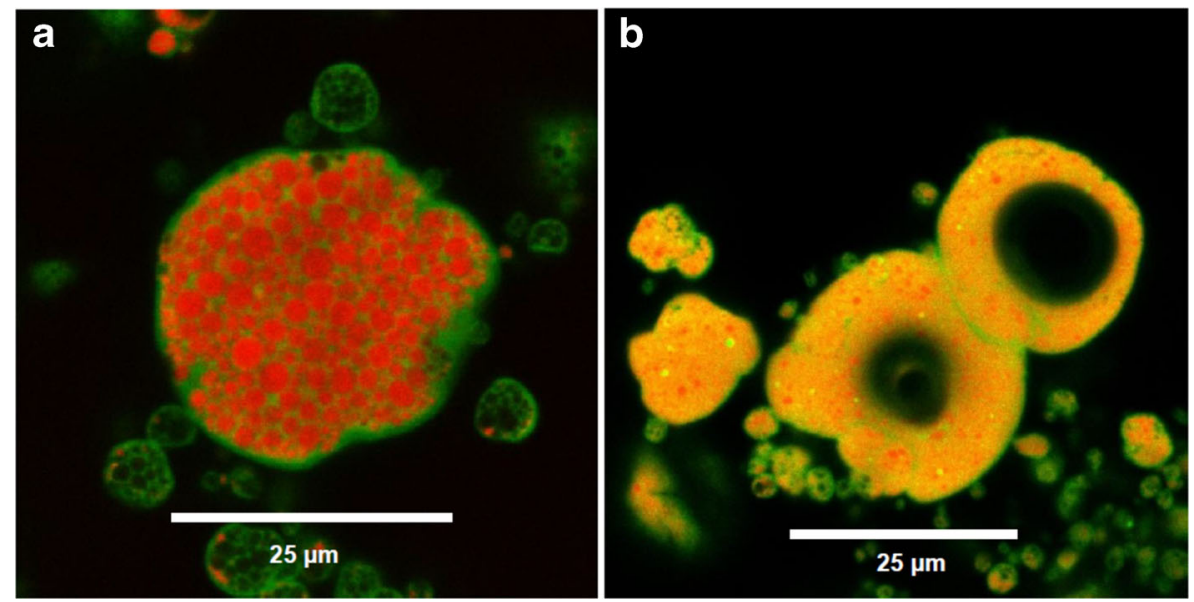

Fig. 8 Confocal micrographs of emulsion powder particles $(20.0 \%, w / w$, soya oil, $12.0 \%, w / w$, maltodextrin) stained with Nile red and Fast green: a $0.5 \%(w / w)$ milk protein concentrate, b $5.0 \%(w / w)$ milk protein concentrate. Red represents the fat phase and green represents proteins. The black hole inside the powder particle is an air vacuole. Scale as shown on the micrographs

aggregates in $\mathrm{CaCas}$ would reduce the emulsifying ability by resisting rearrangement and spreading on the surface of the oil droplet, when compared with MPC-containing emulsions (Euston and Hirst 1999; Ye 2011; Figs. 1, 2, and 3).

The total unadsorbed protein contents were comparable for the MPC and CaCas emulsions at all protein concentrations, except 5.0\% (w/w), when the MPC-containing emulsion had a higher unadsorbed protein concentration (Fig. 5). The specific surface areas of the parent emulsions made using MPC and CaCas were comparable at $5.0 \%$ $(w / w)$ protein concentration (results not shown). However, the protein concentration on the surface of the oil droplets would possibly be higher in the CaCas-containing emulsion than in the MPC-containing emulsion because of the preferential adsorption of aggregated casein material at the surface of the oil droplets in CaCas-containing emulsions and the larger overall average size of these casein aggregates compared with the aggregated species in MPC-containing emulsions (Srinivasan et al. 2001; Ye 2011).

In our previous study, emulsions made using NaCas and WPI showed overlapping distributions of the parent and redispersed emulsions at protein concentrations above $2.0 \%(w / w)$. Below this concentration, the droplet size distribution showed a shift toward a larger size range in both types of emulsion. The MPC- and CaCas-containing emulsions showed similar redispersion behavior, although the average droplet diameters in the MPC and CaCas emulsions were larger than those in the WPI and NaCas emulsions at a given protein concentration (Figs. 1, 2, and 3). In addition, the minimum concentration of protein that was required to produce emulsions that were stable during the spray drying process was $3.0 \%(w / w)$, compared with $2.0 \%(w / w)$ for the NaCasand WPI-containing emulsions (Taneja et al. 2013). It was suggested that the amount of unadsorbed protein in the bulk phase of the parent emulsions might play a crucial role in stabilizing the oil droplets during spray drying. Protein molecules, because of their high surface activity, are likely to migrate to the newly created air-water interface during spray drying (Taneja et al. 2013; Vega and Roos 2006). When the surface of the oil droplet is saturated with protein molecules and the bulk phase of the emulsion has 
sufficient unadsorbed protein, the oil droplet is stable during drying. However, for emulsions with a low concentration of unadsorbed protein in the bulk phase $(\leq 1.0 \%$ for WPI or NaCas emulsions), protein molecules could potentially migrate from the surface of the oil droplet to the air-water interface, causing "gaps" in the oil droplet interface and leading to coalescence and/or bridging flocculation (Taneja et al. 2013). The concentration of non-aggregated unadsorbed protein molecules would effectively be lower in emulsions made using MPC and CaCas because of the presence of aggregated species. In addition, casein aggregates are thought to be less surface active than nonaggregated caseins (Ye et al. 2000). Therefore, a higher concentration of protein in the bulk phase would be required in MPC and CaCas emulsions to stabilize the oil droplets during drying, compared with $\mathrm{NaCas}$ and WPI emulsions, as seen in our results.

Even though the emulsions made using MPC and $\mathrm{CaCas}$ showed good redispersion behavior above a protein concentration of $3.0 \%(w / w)$, in general, the CaCas-containing emulsions had higher average droplet diameters than the MPC-containing emulsions at similar protein concentrations (Figs. 1, 2, and 3). This was possibly due to the larger aggregated species on the surface of the emulsions containing CaCas compared with the relatively smaller sized aggregated species and the non-aggregated species in the emulsions containing MPC. These larger aggregated species are likely to respond relatively more slowly to redistribution of the adsorbed layer during spray drying because of their comparatively lower hydrophobicity and flexibility, resulting in recoalescence (Vega and Roos 2006; Vega et al. 2007).

The focus of this study was on the importance of droplet stability during spray drying, as it is essential that powdered emulsions reconstitute to the same droplet size distribution as in the parent emulsions. The presence of free oil on the surface of powder renders the powder particles hydrophobic, drastically reducing their solubility in water (Nijdam and Langrish 2006). In addition, it has been shown that oil on the surface allows the formation of weak bridges between particles, leading to a loss of quality through caking. The phenomenon of caking involves the transformation of a free-flowing powder into lumps and ultimately into an agglomerated solid, leading to a loss of functionality, i.e., wettability, flowability, oxidative stability, etc. (Vega and Roos 2006).

The solvent extraction method recovers both oil on the surface and some oil from inside the powder through cracks and pores (Vega and Roos 2006). Therefore, the extractable oil value potentially reflects the oil-retaining capabilities of the powdered emulsions as well as the presence of oil on the surface. The oil on the surface is thought to originate from the emulsion droplets entering the air-water interface and opening at the surface during drying while water is being removed from the drying droplet (Hotrum et al. 2002). However, if sufficient protein were available in the bulk phase of the emulsion during drying, the opening of the emulsion droplets would be limited, as proteins would dominate the surface. The decrease in the amount of extractable oil for the MPC-containing emulsions was possibly indicative of this behavior (Fig. 6). At MPC concentrations of 3.0 and $5.0 \%(w / w)$ in the emulsions, as the extractable oil value was below $100 \mathrm{mg} \cdot \mathrm{g}^{-1}$, less agglomeration was seen in the electron micrographs (Fig. 7). In contrast, at lower protein concentrations in the emulsion, the powder particles were highly agglomerated and had higher extractable oil values. At all protein concentrations, the CaCas-containing powdered emulsions had higher levels of extractable oil than the MPC-containing powdered emulsions. At 5.0\% $(w / w)$ protein

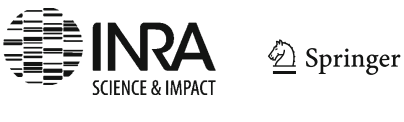


in the emulsion, the electron micrographs did not show "molten" agglomerated particles in the powdered emulsions containing CaCas even though the extractable oil at this concentration was much greater than that in the powdered emulsions made using $0.5 \%$ $(w / w)$ MPC, which showed extensive agglomeration. It seems possible that the powdered emulsions made with $\mathrm{CaCas}$ were of a highly porous nature and were more pervious to solvent than the powdered emulsions made with MPC.

In conclusion, the composition of the parent emulsion plays a significant role in the stability of oil-in-water emulsion during the spray drying process and would most likely impact basic end-user or consumer properties, e.g., redispersion. This work provided further the understanding of the mechanisms behind stabilization of milk proteinstabilized emulsions with high oil content during the drying process and may support the development of value-added dairy-based powdered emulsions.

Acknowledgments The authors thank Dr. Jianyu Chen and Mr. Doug Hopcroft (Manawatu Microscopy and Imaging Centre, Massey University, Palmerston North) for their assistance with confocal and electron microscopy, respectively.

\section{References}

Dickinson E (1999) Caseins in emulsions: interfacial properties and interactions. Int Dairy J 9:305-312

Dybowska BE (2008) Properties of milk protein concentrate stabilized oil-in-water emulsions. J Food Eng 88: $507-512$

Euston SR, Hirst RL (1999) Comparison of the concentration-dependent emulsifying properties of protein products containing aggregated and non-aggregated milk protein. Int Dairy J 9:693-701

Faldt P, Bergenstahl B (1996) Spray-dried whey protein/lactose/soybean oil emulsions. 1. Surface composition and particle structure. Food Hydrocoll 10:421-429

Farrer D, Lips A (1999) On the self assembly of sodium caseinate. Int Dairy J 9:281-286

Havea P (2006) Protein interaction in milk protein concentrate powders. Int Dairy J 16:415-422

Hotrum NE, van Vliet T, Stuart MAC, van Aken GA (2002) Monitoring entering and spreading of emulsion droplets at an expanding air/water interface: a novel technique. J Colloid Interface Sci 247: $125-131$

Jafari SM, He YH, Bhandari B (2007) Effectiveness of encapsulating biopolymers to produce sub-micron emulsions by high energy emulsification techniques. Food Res Int 40:862-873

Kelly P (2011) In: Fuquay JW, Fox PF, McSweeney PLH (eds) Encyclopedia of dairy sciences. Elsevier Inc., Amsterdam

Kim EHJ, Chen XD, Pearce D (2005) Effect of surface composition on the flowability of industrial spray-dried dairy powders. Colloids Surf B 46:182-187

Kim EHJ, Chen XD, Pearce D (2009) Surface composition of industrial spray-dried milk powders. 1. Development of surface composition during manufacture. J Food Eng 94:163-168

Lucey JA, Srinivasan M, Singh H, Munro P (2000) Characterization of commercial and experimental sodium caseinate by multiangle laser light scattering and size exclusion chromatography. J Agric Food Chem 48: 1610-1616

Murrieta-Pazos I, Gaiani C, Galet L, Calvet R, Cuq B, Scher J (2012) Food powders: surface and form characterization revisited. J Food Eng 112:1-21

Nijdam JJ, Langrish TAG (2006) The effect of surface composition on the functional properties of milk powders. J Food Eng 77:919-925

Sanchez CC, Patino JMR (2005) Interfacial, foaming and emulsifying characteristics of sodium caseinate as influenced by protein concentration in solution. Food Hydrocoll 19:407-416

Singh H (2005) In: Dickinson E (ed) Food colloids: interactions, microstructure and processing. Royal Society of Chemistry, Cambridge

Singh H, Ye A (2009) In: Thompson A, Boland M, Singh H (eds) Milk proteins: from expression to foods. Elsevier Inc., London 
Srinivasan M, Singh H, Munro PA (1999) Adsorption behavior of sodium and calcium caseinates in oil-inwater emulsions. Int Dairy J 9:337-341

Srinivasan M, Singh H, Munro PA (2001) Creaming stability of oil-in-water emulsions formed with sodium and calcium caseinates. J Food Sci 66:441-446

Srinivasan M, Singh H, Munro PA (2003) Influence of retorting (121 ${ }^{\circ} \mathrm{C}$ for $15 \mathrm{~min}$ ), before or after emulsification, on the properties of calcium caseinate oil-in-water emulsions. Food Chem 80:61-69

Taneja A, Ye A, Jones JR, Archer R, Singh H (2013) Behaviour of oil droplets during spray drying of milkprotein-stabilised oil-in-water emulsions. Int Dairy J 28:15-23

Vega C, Roos YH (2006) Invited review: Spray-dried dairy and dairy-like emulsions - compositional considerations. J Dairy Sci 89:383-401

Vega C, Goff DH, Roos YH (2007) Casein molecular assembly affects the properties of milk fat emulsions encapsulated in lactose or trehalose matrices. Int Dairy J 17:683-695

Ye AQ (2008) Interfacial composition and stability of emulsions made with mixtures of commercial sodium caseinate and whey protein concentrate. Food Chem 110:946-952

Ye AQ (2011) Functional properties of milk protein concentrates: emulsifying properties, adsorption and stability of emulsions. Int Dairy J 21:14-20

Ye AQ, Srinivasan M, Singh $\mathrm{H}$ (2000) Influence of $\mathrm{NaCl}$ addition on the properties of emulsions formed with commercial calcium caseinate. Food Chem 69:237-244 